\title{
Secoiridoids delivered as olive leaf extract induce acute improvements in human vascular function and reduction of an inflammatory cytokine: a randomised, double-blind, placebo-controlled, cross-over trial
}

\author{
Stacey Lockyer ${ }^{1}$, Giulia Corona ${ }^{1,2}$, Parveen Yaqoob $^{1}$, Jeremy P. E. Spencer ${ }^{1 *}$ and Ian Rowland ${ }^{1}$ \\ ${ }^{1}$ Hugh Sinclair Unit of Human Nutrition, Department of Food and Nutritional Sciences, University of Reading, \\ Berkshire RG6 GAP, UK \\ ${ }^{2}$ Life Sciences Department, Health Sciences Research Centre, Whitelands College, University of Roehampton, \\ Holybourne Avenue, London SW15 4JD, UK
}

(Submitted 19 July 2014 - Final revision received 20 March 2015 - Accepted 25 March 2015 - First published online 8 June 2015)

\section{Abstract}

The leaves of the olive plant (Olea europaea) are rich in polyphenols, of which oleuropein and hydroxytyrosol (HT) are most characteristic. Such polyphenols have been demonstrated to favourably modify a variety of cardiovascular risk factors. The aim of the present intervention was to investigate the influence of olive leaf extract (OLE) on vascular function and inflammation in a postprandial setting and to link physiological outcomes with absorbed phenolics. A randomised, double-blind, placebo-controlled, cross-over, acute intervention trial was conducted with eighteen healthy volunteers (nine male, nine female), who consumed either OLE ( $51 \mathrm{mg}$ oleuropein; $10 \mathrm{mg}$ HT), or a matched control (separated by a 4-week wash out) on a single occasion. Vascular function was measured by digital volume pulse (DVP), while blood collected at baseline, 1, 3 and $6 \mathrm{~h}$ was cultured for $24 \mathrm{~h}$ in the presence of lipopolysaccharide in order to investigate effects on cytokine production. Urine was analysed for phenolic metabolites by HPLC. DVP-stiffness index and ex vivo IL-8 production were significantly reduced $(P<0 \cdot 05)$ after consumption of OLE compared to the control. These effects were accompanied by the excretion of several phenolic metabolites, namely HT and oleuropein derivatives, which peaked in urine after $8-24 \mathrm{~h}$. The present study provides the first evidence that OLE positively modulates vascular function and IL-8 production in vivo, adding to growing evidence that olive phenolics could be beneficial for health.

Key words: Olive leaf extract: CVD: Vascular function: Inflammatory cytokines: Dietary polyphenols bioavailability

Consumption of a 'Mediterranean diet' has been associated with increased longevity and a decreased risk of chronic diseases, including CVD, compared with other types of $\operatorname{diet}^{(1)}$. These effects are attributed, in part, to the olive oil (OO) component of the diet ${ }^{(2)}$. Indeed, research comparing refined $v$. extra virgin $\mathrm{OO}$ has highlighted the biological effects of the polyphenols contained within the water-soluble fraction $^{(3)}$, in addition to other biologically active compounds such as phytosterols and lipid-soluble vitamins. The presence of these compounds in OO may underpin a degree of its health-promoting properties, relative to similar MUFA-rich oils $^{(4)}$. Compared to OO, the leaves of the olive plant (Olea europaea) contain similar phenolics, although at significantly higher concentrations than that of the olive fruit and derived oil (1450 mg total phenolics/100g fresh leaf ${ }^{(5)} v .110 \mathrm{mg} /$ $100 \mathrm{~g}$ fruit $^{(6)}$ and $23 \mathrm{mg} / 100 \mathrm{ml}$ extra virgin $\mathrm{OO}^{7}$ ). The most abundant phenolic compounds present in the leaves are verbascoside, apigenin-7-glucoside, luteolin-7-glucoside, hydroxytyrosol (HT), tyrosol and the secoiridoid, oleuropein (see Fig. 1), with secoiridoids being uniquely present in plants of the Olearaceae family ${ }^{(8)}$.

Anecdotally, olive leaf has been reported useful in treating a range of medical conditions ${ }^{(9)}$. Animal and in vitro data have suggested a wide array of bioactive properties for phenolic-rich olive leaf extract (OLE), including hypotensive ${ }^{(10)}$, antioxidant $^{(11)}$, anti-inflammatory ${ }^{(12)}$, hypoglycaemic ${ }^{(11)}$, lipidlowering $^{(13)}$ and vasoactive ${ }^{(14)}$ effects, many of which have been successfully replicated in human studies, leading to the conclusion that chronic OLE supplementation has the potential to modify CVD risk (for a review see Lockyer et $a l .{ }^{(15)}$ ). Phenolicrich $\mathrm{OO}$ has been reported to improve vascular function relative to phenolic-poor OO, postprandially ${ }^{(16,17)}$. However,

Abbreviations: DVP, digital volume pulse; DVP-SI, digital volume pulse-stiffness index; EA, elenolic acid; EDA, elenolic acid dialdehyde; HT, hydroxytyrosol; HValc, homovanillic alcohol; OLE, olive leaf extract; OO, olive oil; RT, retention time.

*Corresponding author: Professor J. P. E. Spencer, fax +44 118931 0080, email j.p.e.spencer@reading.ac.uk 
<smiles>C/C=C1/C(CC(=O)OCCc2ccc(O)c(O)c2)C(C(=O)OC)=CO[C@@H]1OC1OC(CO)[C@@H](O)[C@H](O)[C@H]1O</smiles>

Apigenin-7-glucoside<smiles>CC(C)=CC(=O)O[C@H]1C(CO)OC(OCCc2ccc(O)c(O)c2)C(O)C1OC1C[C@H](C)[C@@H](O)C(O)[C@H]1O</smiles>

Fig. 1. Most abundant phenolics present in olive leaf.

data pertaining to the influence of OLE on vascular function are currently lacking. In the present study, we address this by determining the extent to which OLE intake influences two measures of CVD risk ${ }^{(18)}$ : arterial stiffness (assessed using digital volume pulse (DVP)) and inflammatory status in a postprandial setting. With respect to the latter, acute intake of $O O$, with a high phenolic content, has been shown to repress the expression of several pro-inflammatory genes in vivo, including the cytokines IL-6 and IL-1 $\beta^{(19)}$, though knowledge regarding the effects of OLE on inflammation is very limited.

Notably, if phenolic components are to mediate the actions of OO and OLE on vascular function and inflammatory processes in vivo, it is necessary that they are absorbed into the circulation. While absorption of phenolics following $\mathrm{OO}$ intake has been extensively explored ${ }^{(20)}$, only two studies $^{(21,22)}$ using OLE have been conducted, and direct comparisons of specific absorption/metabolism profiles between OLE and OO cannot be made because of the presence of different phenolics, their relative concentrations and food matrix effects ${ }^{(23)}$. As such, in the present study, we aim to assess the absorption and metabolism of polyphenols from
OLE, in addition to the physiological outcomes, in order to build a greater understanding of the 'cause-and-effect' relationship between OLE intake and potential biological effects.

\section{Experimental methods}

\section{Study population}

Healthy male and female subjects were recruited from the University of Reading and the surrounding area via email and poster advertisements in May 2011. Smokers, those with alcohol consumption of more than 21 units/week, or those with any dietary restrictions, including vegetarians and those on weight-reduction diets, were excluded. Individuals who had suffered significant illness in the previous 12 months, or had gastrointestinal, blood-clotting or metabolic disorders, and females who were pregnant or breastfeeding were also excluded. Subjects had not taken antibiotics in the previous 3 months and were not taking any lipid-modifying or blood-clotting medication, vitamin, mineral and/or fish oil 
supplements at the time of completing the study. Potential subjects underwent a screening process and individuals with blood pressure $>150 / 90 \mathrm{mmHg}$; $\mathrm{Hb}<125 \mathrm{~g} / \mathrm{l}$ for men and $<110 \mathrm{~g} / 1$ for women; $\gamma$-glutamyl transferase $>1.3 \mu \mathrm{kat} / 1$ or cholesterol $>6.5 \mathrm{mmol} / \mathrm{l}$ were excluded from the study. Twenty suitable subjects were identified and recruited into the study. Two subjects completed one study visit and were lost to follow up; therefore, eighteen subjects completed the study. Based on a medium effect size with respect to stiffness index (a difference of $0.30 \mathrm{~m} / \mathrm{s}$ ) and population standard deviation $(0.30 \mathrm{~m} / \mathrm{s})$, we retrospectively calculate that a sample size of fifteen participants would provide considerable power (0.97); therefore, we conclude that our sample size of eighteen provides ample statistical power for detecting a change in digital volume pulse-stiffness index (DVP-SI). Baseline characteristics of the subjects are detailed in online Supplementary Table S1.

\section{Study design}

The study was a double-blind, randomised, controlled, crossover trial (Clinicaltrials.gov ID: NCT01479699) conducted according to the guidelines laid down in the Declaration of Helsinki. All procedures involving human subjects were approved by the University of Reading Research Ethics Committee (UREC 11/30). Written, informed consent was obtained from all subjects. Eighteen subjects (nine male, nine female) aged 19-40 years completed two clinical visits at the Hugh Sinclair Nutrition Unit, University of Reading, approximately 4 weeks apart between June and September 2011. Female subjects completed both visits during the same phase of their menstrual cycle in an attempt to control for any possible effects of hormones on vascular function ${ }^{(24)}$. Subjects were asked to consume a low polyphenol diet (devoid of tea, coffee, fruit, vegetables, alcoholic beverages, cocoa, wholegrain and avoiding all olive-containing products in particular) for $24 \mathrm{~h}$ before the start of each study day and remained on this diet until after the last urine sample was collected. Compliance was confirmed by the HPLC analysis of baseline urine samples. Subjects arrived at the clinical unit in a 12-h fasted state. A cannula was inserted into the arm and a baseline blood sample collected. Subjects ingested either four capsules containing OLE or four control capsules as suggested by the product manufacturer. Blood samples were collected at baseline and 1, 3 and $6 \mathrm{~h}$ after capsule ingestion. Vascular function was measured by PCA2 Pulse Trace (Micromedical) to obtain DVP at baseline and 0.5, 1, 1.5, 2, 3, 4, 6 and $8 \mathrm{~h}$ after capsule ingestion. Urine was collected at baseline, 0-4, 4-8 and 8-24 h after capsule consumption. Samples were centrifuged at $1800 \mathrm{~g}, 4^{\circ} \mathrm{C}$ for $10 \mathrm{~min}$ and frozen at $-80^{\circ} \mathrm{C}$ until analysis. The total volume of urine produced during each time period was recorded. Subjects were provided with a standard lowfat lunch of low phenolic content (one ham sandwich and one low-fat cheese sandwich on white bread with very-lowfat margarine and a very-low-fat yoghurt) after the 4-h sample collection and a meal of low phenolic content (chicken, ham and mashed potato) after the 8-h samples.
Low-nitrate mineral water (Buxton) was provided to subjects during the whole intervention period as required.

\section{Test products}

Four OLE capsules contained a total of $1600 \mathrm{mg}$ OLE (400 mg per capsule in $672.5 \mathrm{mg}$ safflower oil) delivering a total of $51.12 \mathrm{mg}$ oleuropein and $9.67 \mathrm{mg}$ HT, as verified by two independent laboratories at Southern Cross University, Australia, and The Cawthron Institute, New Zealand. The full phenolic profile can be found in Table 1. Control capsules each contained $900 \mathrm{mg}$ safflower oil and were identical in size and appearance to OLE capsules. All capsules were provided by Comvita New Zealand Limited and product safety was assessed as previously detailed ${ }^{(25)}$

\section{Measurement of vascular function}

Subjects were asked to rest supine during DVP measurements. The DVP was obtained by photoplethysmography of the index finger using the PulseTrace PCA2 system to calculate stiffness index (DVP-SI). The Pulse Trace is operator independent and very reproducible. Stiffness index has been found to be positively correlated with cardiovascular risk factors including age, blood pressure, waist:hip ratio and carotid thickness ${ }^{(26)}$. Stiffness index values are sensitive to small changes in vascular tone induced by vasodilators ${ }^{(27)}$ and are strongly correlated with central pulse-wave velocity, a recognised measure of arterial stiffness.

\section{Urinary phenolic analysis}

Bioavailability of phenolic compounds present in OLE was assessed by HPLC analysis of urine. Before analysis, the samples were subjected to $\beta$-glucuronidase treatment (an enzyme that also has sulfuronidase activity) in order to cleave glucuronide and sulphate moieties, yielding polyphenol aglycones for detection by HPLC. As such, peaks identified by HPLC represent the sum of excretion of pure compounds and all conjugates (e.g. the presence of HT aglycone and any HT glucuronides and sulphates would be indicated by the HT peak). After centrifugation ( $2 \mathrm{~min}$ at $13000 \mathbf{g}$ ), urine samples were mixed in a $1: 1$ ratio with $0.05 \mathrm{M}$-ammonium

Table 1. Phenolic content of olive leaf extract capsules

\begin{tabular}{lc}
\hline Compound & Content in four capsules $(\mathrm{mg})$ \\
\hline Oleuropein & 51.124 \\
Hydroxytyrosol & 9.666 \\
Verbascoside & 0.344 \\
Luteolin & 0.249 \\
Phenolic acids* & 0.233 \\
Rutin & 0.150 \\
Apigenin & 0.046 \\
Quercetin & 0.038 \\
Flavonoids & 0.028 \\
Kaempferol & 0.021 \\
\hline
\end{tabular}

${ }^{*}$ Calculated as caffeic acid 
acetate buffer adjusted to $\mathrm{pH} 5$ with acetic acid (final volume $0.8 \mathrm{ml}$ ). $\beta$-Glucuronidase $(50 \mathrm{mg} ;$ Helix pomatia; Sigma Aldrich) in $500 \mu \mathrm{l}$ sodium acetate buffer $(0 \cdot 1 \mathrm{~m})$ was added to the samples, which were then vortexed before incubation at $37^{\circ} \mathrm{C}$ for $90 \mathrm{~min}$. After incubation, $10 \mu \mathrm{l}$ acetic acid was added to the samples on ice. The samples were centrifuged again at $13000 \mathrm{~g}$ for $2 \mathrm{~min}$ and $0.6 \mathrm{ml}$ distilled water was added before solid-phase extraction using a vacuum manifold with Strata C18-E ( $55 \mu \mathrm{m}, 70 \mathrm{~A})$ cartridges (Phenomenex). The cartridges were conditioned with $1 \mathrm{ml}$ methanol and equilibrated with $2 \mathrm{ml} 0.05 \mathrm{M}$-ammonium acetate solution before sample loading and separation under gravity. To elute phenolics, the cartridges were washed with $1 \mathrm{ml} 0.05 \mathrm{M}$-ammonium acetate buffer and dried under vacuum for $5 \mathrm{~min}$. Collection tubes were changed and $2.5 \mathrm{ml}$ ethyl acetate was added to each cartridge. The collection tubes were taken to complete dryness using a Speedvac system (Thermo Fisher Scientific, Inc.). Dried extracts were reconstituted in $125 \mu \mathrm{l}$ mobile phase A (95\% water, 5\% methanol, $0 \cdot 1 \% 5 \mathrm{~m}-\mathrm{HCl})$ and vortexed for $5 \mathrm{~min}$. The samples were centrifuged for $2 \mathrm{~min}$ at $13000 \mathrm{~g}$, and finally the supernatants were added to the HPLC vials.

HPLC analysis was performed to characterise and quantify the identifiable phenolic components. Precisely $50 \mu \mathrm{l}$ of the extracted urine sample was injected into the HPLC system. Separation of phenolic constituents was achieved using reverse-phase HPLC using a Nova-Pak C18 column $(4.6 \times 250 \mathrm{~mm})$ (Waters) with $4 \mu \mathrm{m}$ particle size. A Waters 626 pump and 600-controller system with an auto-injector 717 and a photodiode array detector 996 linked to the Millennium Software system was utilised. The temperature of the column was maintained at $30^{\circ} \mathrm{C}$. The mobile phase consisted of A: $95 \%$ water, $5 \%$ methanol, $0 \cdot 1 \% 5 \mathrm{~m}-\mathrm{HCl}$ and $\mathrm{B}: 50 \%$ acetonitrile, $50 \%$ water and $0 \cdot 1 \% \mathrm{HCl}$ and was pumped through the column at $0.7 \mathrm{ml} / \mathrm{min}$. The following gradient system was used (min/\% B): 0/5, 50/100, 60/100, 65/5, for detection of all compounds. The eluant was monitored by photodiode array detection at $280 \mathrm{~nm}$ and the spectra of products were obtained over the $220-600 \mathrm{~nm}$ range. The following pure standard compounds were purchased from Extrasynthese: oleuropein, HT, tyrosol, homovanillic alcohol (HValc). 3,4-Dihydroxyphenylethanol-elenolic acid (EA, oleuropein aglycone) and 3,4-dihydroxyphenylethanol-elenolic acid dialdehyde (EDA, oleuropein aglycone di-aldehyde) were obtained as previously described by our group ${ }^{(28)}$. Calibration curves were constructed using authentic standards $(0-100 \mu \mathrm{M})$, and in each case were found to be linear with correlation coefficients of $>0.99$. Compounds identified in the samples were characterised by retention times (RT) and spectral similarity to standards. Compounds identified in urine were quantified after adjustment for volume of urine produced.

\section{Whole blood culture}

Blood was collected into heparin tubes (Greiner BioOne Limited) at baseline and 1,3 and $6 \mathrm{~h}$ after capsule consumption. These time points were selected in an attempt to capture probable peak phenolic concentrations in plasma ${ }^{(22)}$. Whole blood was immediately diluted $1 / 1$ with Roswell Park Memorial
Institute 1640 medium (Lonza Wokingham Limited), supplemented with $1 \%$ antibiotics (Penicillin 10.000 UI/ml streptomycin $10 \cdot 000 \mathrm{UI} / \mathrm{ml}$ Lonza Wokingham Limited). Precisely $1.8 \mathrm{ml}$ diluted blood was added to one well in a twentyfour-well plate. Lipopolysaccharide was added to give a final concentration of $1 \mu \mathrm{g} / \mathrm{ml}$. An additional well containing $1.8 \mathrm{ml}$ diluted blood and $0.2 \mathrm{ml}$ medium served as a control. The plates were incubated at $37^{\circ} \mathrm{C}$ in a $5 \% \mathrm{CO}_{2}$ atmosphere for $24 \mathrm{~h}$. After incubation, the contents of the well were removed and centrifuged at $1000 \mathrm{~g}$ for $2 \mathrm{~min}$. Supernatants were collected and stored at $-80^{\circ} \mathrm{C}$ until analysis.

\section{Cytokine analysis}

IL-1 $\beta$, IL-6, IL-10, TNF- $\alpha$ and IL-8 concentrations were measured in whole blood culture supernatants using a Human base kit Panel A Fluorokine MAP (Multi-analyte Profiling) ELISA (R\&D Systems Europe Limited) and a Luminex 200 Analyser (Invitrogen) following standard instructions provided by the manufacturer.

\section{Statistical analysis}

Prism (GraphPad Software) was used to analyse the data. Data were tested for normality using a $K-S$ test. Differences in DVP-SI, IL-1 $\beta$, IL-6, TNF- $\alpha$, IL- 8 and IL-10 in ex vivo whole blood cultures by treatment were identified using a two-way ANOVA with repeated measures. Post hoc analysis was subjected to Bonferroni correction. $P$ values less than 0.05 were treated as significant.

\section{Results}

\section{Vascular function}

The stiffness index (DVP-SI) changed significantly from baseline over the course of the study day (significant time effect,

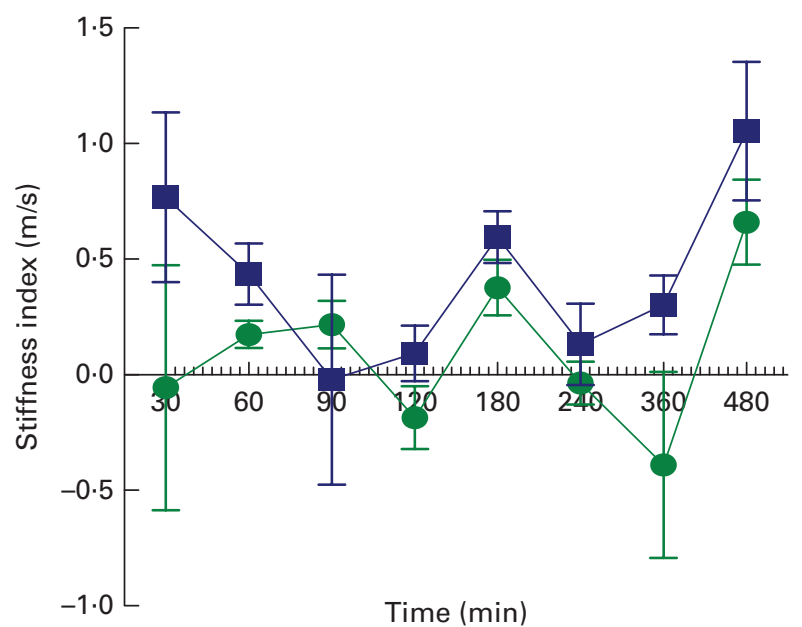

Fig. 2. Effect of olive leaf extract (OLE) on digital volume pulse-stiffness index. Values are mean changes from baseline with their standard errors represented by vertical bars $(n 18)$ after ingestion of $1600 \mathrm{mg}$ OLE $(--)$ or a control (-) on a single occasion. Two-way ANOVA with repeated measures revealed a significant treatment effect $(P=0.0085)$ and a time effect $(P=0.0028)$. A colour version of this figure can be found online at http://www. journals.cambridge.org/bjn 
$F_{1,7}=3 \cdot 23 ; P=0 \cdot 0028$, Fig. 2$)$. In addition, there was a highly significant treatment effect on DVP-SI $\left(F_{1,7}=7 \cdot 05 ; P=0.0085\right)$, with means of $0.76(\operatorname{sem} 1.63) \mathrm{m} / \mathrm{s}$ for the control group and $3.36($ SEM 1.79$) \mathrm{m} / \mathrm{s}$ for the OLE group over the course of the trial, indicating that arterial stiffness was significantly lower over the study day after consumption of the OLE capsules compared with the control capsules. However, post boc analysis indicated no interaction between DVP-SI and treatment at any individual time point. In addition, there was a trend for OLE to attenuate the post-meal increase in arterial stiffness (between 240 and $480 \mathrm{~min}$ ) ( $P=0.074$ ) (Fig. 2).

\section{Ex vivo cytokine production}

Ex vivo, lipopolysaccharide-stimulated IL-8 production in whole blood cultures (isolated from human participants on the study) was significantly lower $\left(F_{1,2}=4.7 ; \quad P=0.0326\right.$; Fig. 3) after OLE consumption (7368 (SEM 856) pg/ml) compared to the control (15200 (sem 1756) pg/ml). Post boc analysis revealed no interaction between treatment and any individual time point. There was no significant effect of treatment on ex vivo production of IL-1 $\beta$, IL- 6 , TNF- $\alpha$ or IL-10 (data not shown).

\section{Bioavailability}

Analysis of urine samples by HPLC indicated the presence of HT (RT: $8.6 \mathrm{~min}$ ), tyrosol (RT: $14.0 \mathrm{~min}$ ), HValc (RT: $17 \cdot 3 \mathrm{~min}$ ), EDA (RT: $30.3 \mathrm{~min}$ ), oleuropein (RT: $38.3 \mathrm{~min}$ ) and EA (RT: $41.3 \mathrm{~min}$ ) following the intake of OLE (for an example chromatogram see online Supplementary Fig. S1). In addition, a number of other secoiridoid peaks were observed, which were likely to have been derived from the metabolism of oleuropein. These compounds (four of which were observed at RT: $31 \cdot 7,34 \cdot 1,37 \cdot 2$ and $38.8 \mathrm{~min}$ (online Supplementary Fig. S1)), were characterised by RT and spectral similarity to either EDA or oleuropein, although their exact identity

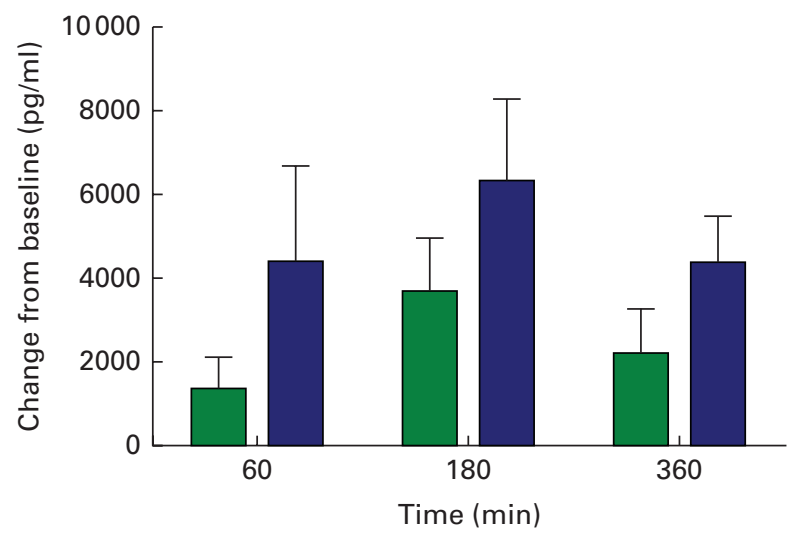

Fig. 3. Effect of olive leaf extract (OLE) on ex vivo lipopolysaccharidestimulated IL-8 production in whole blood cultures. Values are mean changes from baseline with their standard errors represented by vertical bars ( $n$ 16) after ingestion of $1600 \mathrm{mg}$ OLE ( $\square$ ) or a control ( $\square$ ) on a single occasion. Two-way ANOVA with repeated measures revealed a significant treatment effect $(P=0.0326)$. A colour version of this figure can be found online at http://www.journals.cambridge.org/bjn
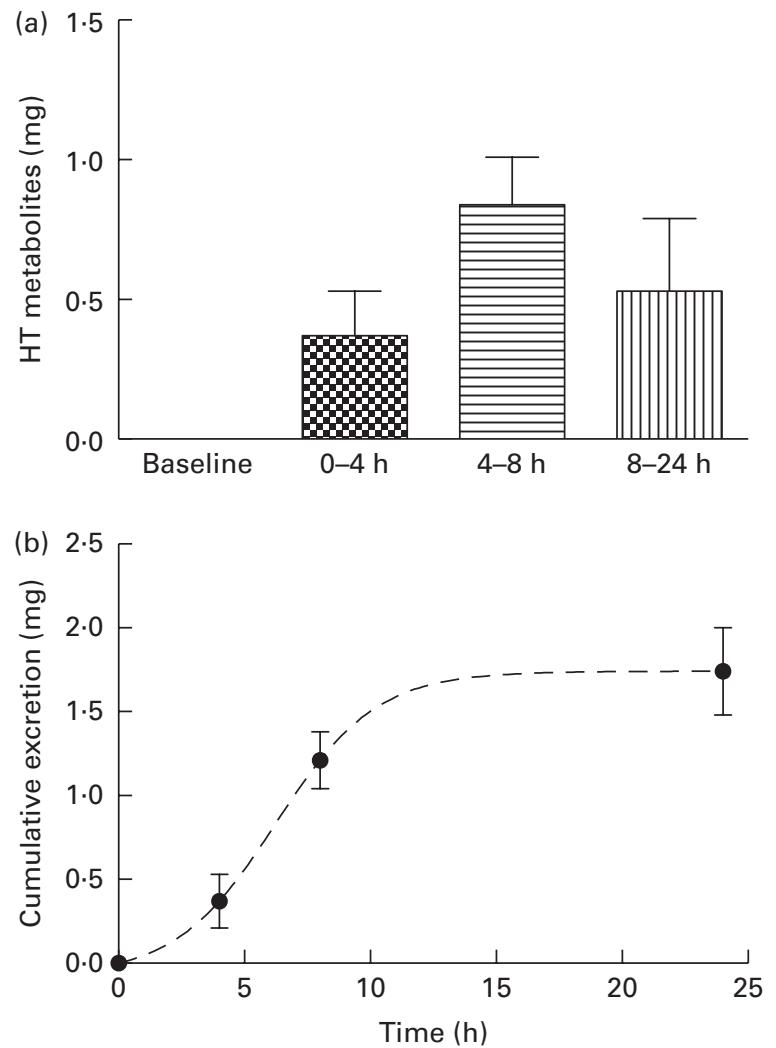

Fig. 4. (a) Time-dependent appearance of hydroxytyrosol (HT) and conjugates in urine and (b) corresponding cumulative excretion $24 \mathrm{~h}$ following ingestion of olive leaf extract (OLE) capsules. Values, derived from HPLC analysis, are means with their standard errors represented by vertical bars ( $n$ 18) after ingestion of $1600 \mathrm{mg}$ OLE, containing $51.12 \mathrm{mg}$ oleuropein and $9.67 \mathrm{mg} \mathrm{HT}$, on a single occasion. $\mathrm{\otimes}$, Baseline; $\mathrm{0}, 0-4 \mathrm{~h}$;, $4-8 \mathrm{~h}$; 两, 8-24h.

remains unconfirmed due to a lack of standards and/or mass spectral data. Compounds derived from oleuropein and eluting between 30 and $60 \mathrm{~min}$, including EA and EDA, were grouped together and tentatively quantified as 'oleuropein equivalents'. HT and its conjugates were identified in urine samples collected at $0-4,4-8$ and $8-24 \mathrm{~h}$, with levels peaking at $4-8 \mathrm{~h}$ (Fig. 4). HValc and its conjugates were found to increase steadily in the urine of subjects between 0 and $8 \mathrm{~h}$, peaking between 8 and $24 \mathrm{~h}$ (Fig. 5). Tyrosol was identified in the urine of one individual (see online Supplementary Fig. S1). Finally, oleuropein equivalents were excreted steadily over the course of the study period, peaking at 8-24 $\mathrm{h}$ following OLE consumption (Fig. 6). The grouped oleuropein metabolites represented the most abundant breakdown product of the OLE capsules. Baseline blood samples were devoid of any compounds of interest.

\section{Discussion}

In the present investigation, we provide evidence that acute consumption of OLE, an alternative source of olive phenolics, improves vascular function and reduces the production of an inflammatory cytokine. These observations are supported by quantitative data regarding the absorption of OLE phenolics, 

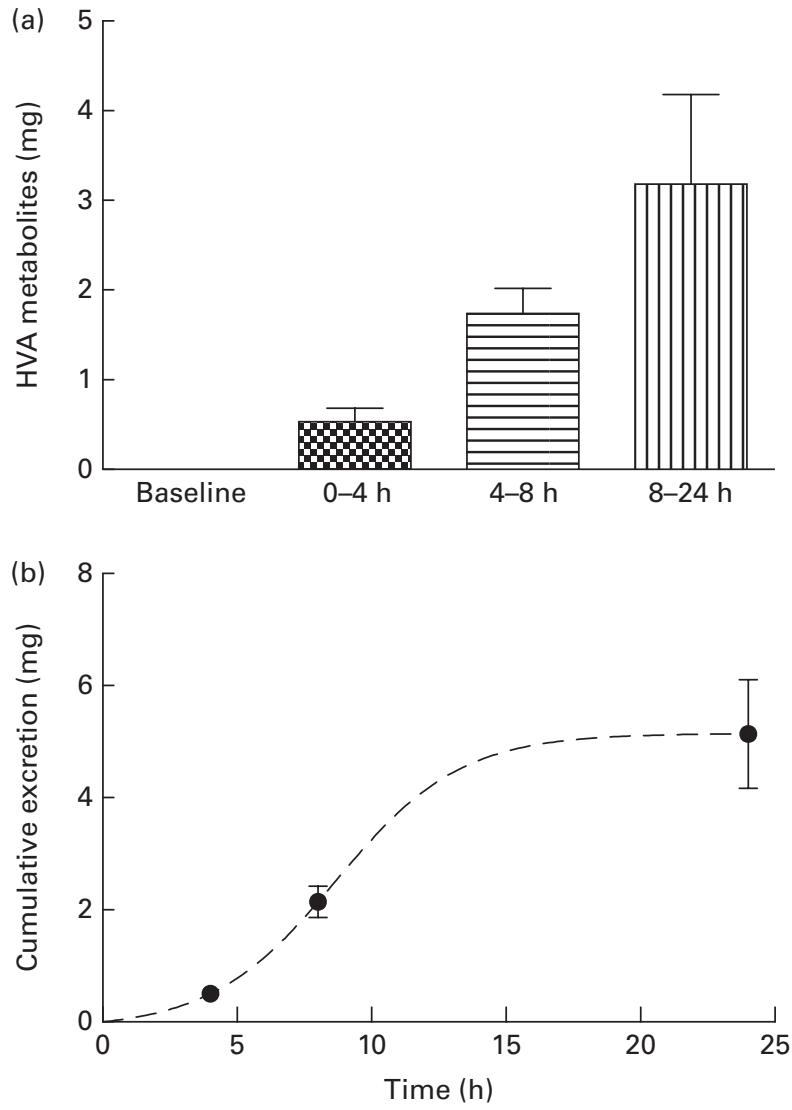

Fig. 5. (a) Time-dependent appearance of homovanillic alcohol (HVA), a breakdown product of hydroxytyrosol, and conjugates in urine and (b) corresponding cumulative excretion $24 \mathrm{~h}$ after ingestion of olive leaf extract (OLE) capsules. Values, derived from HPLC analysis, are means with their standard errors represented by vertical bars $(n 18)$ after ingestion of $1600 \mathrm{mg} \mathrm{OLE}$, containing $51.12 \mathrm{mg}$ oleuropein and $9.67 \mathrm{mg} \mathrm{HT}$, on a single occasion. 因, Baseline; $\mathrm{G}, 0-4 \mathrm{~h} ; \boxminus, 4-8 \mathrm{~h} ; \square, 8-24 \mathrm{~h}$.

indicating the bioavailability of HT, tyrosol, HValc, oleuropein, and for the first time in relation to OLE, EDA and EA and their metabolites in urine. Specifically, OLE led to reduced arterial stiffness, a risk factor and independent predictor for CVD ${ }^{(29)}$ Our finding is in agreement with existing data, indicating that $\mathrm{OO}$ phenolics are capable of improving vascular function $^{(16,17)}$, although the latter study has been called into question because of the criticisms of the methodology ${ }^{(30)}$ and the level of $\mathrm{OO}$ intake $(40 \mathrm{ml})$ used representing a high intake for a single meal ${ }^{(31)}$. The present study intervened with a phenolic-rich OLE supplement, thus avoiding the concurrent delivery of high amounts of fat and energy.

Previous studies have begun to uncover the potential mechanism by which olive and related products may induce vascular improvements. For example, two studies ${ }^{(16,17)}$ observed an increase in plasma nitrates and nitrites, indicating increased levels of NO, a known vasodilator. Although we did not measure NO metabolites in the present study, absorbed phenolic metabolites are thought to influence NO bioavailability via their potential to inhibit NAPDH-oxidase and the production of superoxide ${ }^{(32)}$. In support of this concept, a recent study has reported that acute blueberry intake leads to improved blood flow, which was paralleled by inhibition of neutrophil NADPH oxidase and the appearance of plasma phenolic metabolites ${ }^{(33)}$. Indeed, HT, tyrosol and their metabolites have significant structural homology to the pharmacological NADPH-oxidase inhibitor apocynin ${ }^{(34)}$, and have been proposed as potent NAPDH-oxidase inhibitors in the endothelial cells ${ }^{(32)}$. In the present study, we detected various oleuropein-like metabolites in urine along with HValc and HT in lesser amounts. In addition to supporting the phenolic acid-driven mechanism of vascular effects through NADPH oxidase inhibition, our data suggest that oleuropein may be absorbed, something debated in the previous literature (see online Supplementary Fig. S2) ${ }^{(15)}$. Notably, one study has reported five different glucuronides of oleuropein aglycone in urine (with no HT) ${ }^{(21)}$ and another reporting predominantly glucuronidated HT and negligible oleuropein ${ }^{(22)}$.

The level of oleuropein metabolites identified in urine in the present study is in agreement with a previous ileostomy patient study that reported substantial absorption of OO phenolics $\left(55-66 \%{ }^{(35)}\right)$. In addition, the excretion profile of HT suggests both small $(0-4 \mathrm{~h}$; absorption of free HT present in the OLE) and large ( $4 \mathrm{~h}$ onwards; liberated HT from bacterially metabolised oleuropein) ${ }^{(36)}$. Furthermore, the appearance of HValc is likely to result from the O-methylation of HT by catechol-O-methyltransferase in the large intestinal gut wall
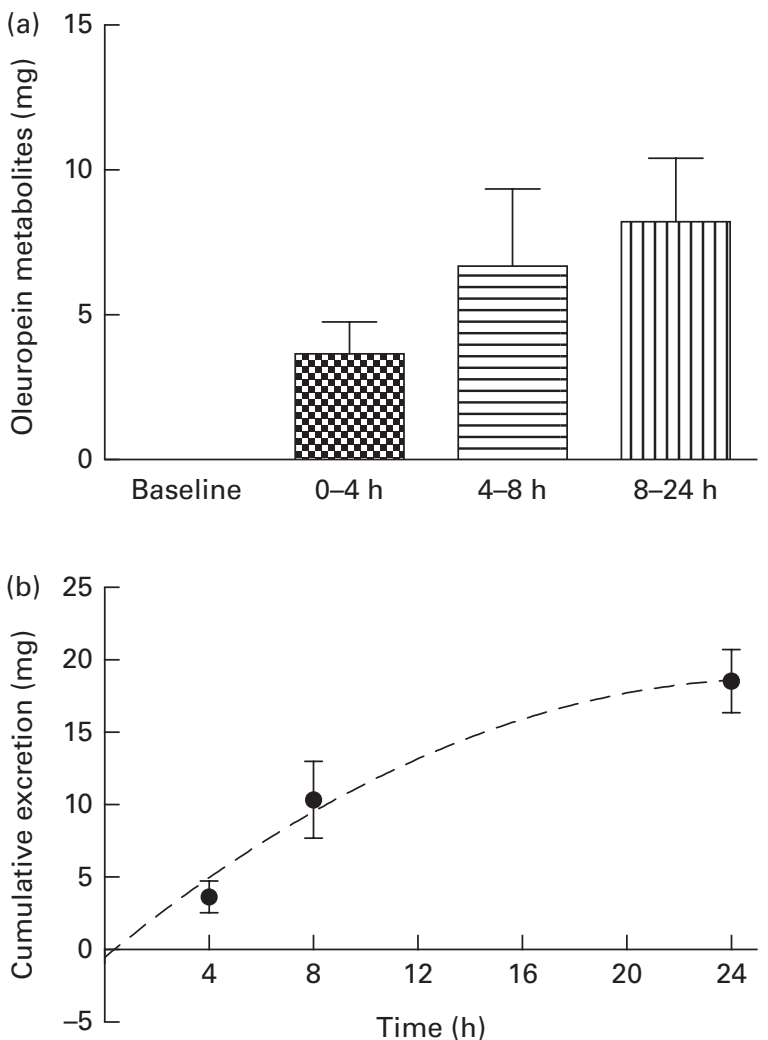

Fig. 6. (a) Time-dependent appearance of oleuropein metabolites in urine and (b) corresponding cumulative excretion, $24 \mathrm{~h}$ following ingestion of olive leaf extract (OLE) capsules. Values, derived from HPLC analysis, are means with their standard errors represented by vertical bars $(n 18)$ after ingestion of $1600 \mathrm{mg}$ OLE, containing $51.12 \mathrm{mg}$ oleuropein and $9.67 \mathrm{mg} \mathrm{HT}$, on a single occasion. $\mathrm{g}$, Baseline; $\mathrm{O}, 0-4 \mathrm{~h}$; $\boxminus, 4-8 \mathrm{~h} ; \mathrm{\square}, 8-24 \mathrm{~h}$. 
and/or the liver. It is possible that two of the unidentified oleuropein-derived compounds peaks are 3,4-DHPEA-EAH ${ }_{2}$ and 3,4-DHPEA-EDAH ${ }_{2}$, which have previously been suggested as potential metabolites of EA and EDA, respectively ${ }^{(37)}$. Only one previous study ${ }^{(22)}$ reports quantitative data regarding OLE metabolites in plasma, suggesting peak plasma concentrations of $0.52 \mathrm{ng} / \mathrm{ml}$ oleuropein and $61 \mathrm{ng} / \mathrm{ml} \mathrm{HT}$ after OLE consumption.

Evidence suggests that lipaemia induced after consumption of high and moderate fat-containing meals causes acute vascular dysfunction ${ }^{(38)}$. In the present study, our intervention meal (containing $7.6 \mathrm{~g}$ fat and $48.5 \mathrm{~g}$ carbohydrate) caused an increased DVP-SI, peaking at $480 \mathrm{~min}$. However, there was a trend in our data suggesting that intake of OLE may attenuate the postprandial vascular impairment. Our data reflect previous observations that phenolic-rich vegetables are capable of attenuating high-fat-meal-induced increases in blood pressure in the presence of L-arginine, thus restoring vascular homeostasis ${ }^{(39)}$. Postprandial lipaemia has also been associated with an increase in circulating inflammatory cytokines ${ }^{(40)}$. In the present study, increases in cytokines were not observed, probably due to the relatively low fat content of the meal. However, postprandial activation and recruitment of neutrophils appears to be associated with a concomitant increase in IL-8, hydroperoxide and endothelial dysfunction ${ }^{(41)}$. As such, the reduction in ex vivo production of IL-8 by an acute dose of OLE may at least partly explain the post-ingestion reduction in DVP-SI.

The anti-inflammatory effects of olive phenolics have been previously indicated $^{(12,13,42)}$, indeed, OLE is used as an ingredient in a patented haemorrhoid treatment ${ }^{(43)}$. In the present study, OLE specifically modulated ex vivo production of IL-8, a low-molecular-weight cytokine produced by a variety of cell types, including monocytes and endothelial cells ${ }^{(44)}$. We suggest that the present ex vivo work, which used samples derived from the postprandial intervention, is reliable as the OLE metabolites generated in vivo were present in the blood used and therefore this is arguably representative of the physiological scenario. It has been postulated that IL-8 plays a key role in the development of atherosclerosis along with other pro-inflammatory cytokines ${ }^{(45)}$. IL-8 may also destabilise existing atherosclerotic plaques by down-regulation of tissue inhibitors of metalloproteinases ${ }^{(46)}$. Although cytokines are not held in the same regard as other biomarkers such as blood pressure and plasma lipids ${ }^{(47)}$, circulating levels of IL-8 have been positively associated with future CVD risk $^{(48)}$. It has been hypothesised that endogenously produced reactive oxygen species directly induce the transcription and translation of IL- $8^{(49)}$. OLE phenolics may selectively inhibit IL-8 production via their redox activity ${ }^{(11)}$, in particular oleuropein and HT, which contain redox-active catechol groups. Green tea catechins and curcumin have also been shown to specifically interfere with $I L-8$ gene expression through inhibition of $\mathrm{NF}-\kappa \mathrm{B}$ activation ${ }^{(50)}$. Similarly, in vitro work has suggested that oleuropein aglycone and HT repress vascular cell adhesion molecule 1 gene transcription by blocking activator protein-1 and NF- $\mathrm{kB}$ activation ${ }^{(51)}$.
The present study provides novel data regarding the positive effects of acute consumption of olive (poly)phenols on the human vasculature. Our data support the concept that OLE positively modulates vascular function and IL-8 production in vivo via the actions of absorbed phenolic metabolites and add to growing evidence that olive phenolics could be beneficial for vascular health. Our data also provide further information relating to the absorption and metabolism of OLE phenolics per se, suggesting that oleuropein metabolites do enter the circulation. One caveat is that the product used has not been completely characterised; in other words, that other bioactives previously identified in OLE, such as minerals, triterpenoids and squalene ${ }^{(52)}$, could have been responsible for the observed response. Nevertheless, the findings presented here add weight to the concept that OLE could represent an important dietary tool in lowering CVD risk.

\section{Supplementary material}

To view supplementary material for this article, please visit http://dx.doi.org/10.1017/S0007114515001269

\section{Acknowledgements}

S. L. would like to thank the following people for their contributions: Kim Jackson and Rada Mihaylova for cannulation of subjects, Rhoyu Zhang and Edna Nyangale for help with implementation of this human study. Angelika Kristek, Doriane Gérard and Amélie Abelard for help with subsequent analytical work.

This work was funded by Comvita Limited and Callaghan Innovation, who supported $50 \%$ of the funding as a Technology for Business Growth grant.

Comvita had no part in the design or running of the study or analysis; therefore, the authors declare no conflict of interest.

The authors' contributions are as follows: S. L., J. P. E. S., P. Y. and I. R. designed the research. S. L. conducted the research. G. C. aided with laboratory and subsequent analytical work. S. L. analysed the data. S. L. wrote the paper with contributions from all authors. J. P. E. S. had primary responsibility for final content.

\section{References}

1. Barzi F, Woodward M, Marfisi R, et al. (2003) Mediterranean diet and all-causes mortality after myocardial infarction: results from the GISSI-Prevenzione trial. Eur I Clin Nutr 57, 604-611.

2. López-Miranda J, Pérez-Jiménez F, Ros E, et al. (2010) Olive oil and health: summary of the II international conference on olive oil and health consensus report, Jaén and Córdoba (Spain) 2008. Nutr Metab Cardiovasc Dis 20, 284-294.

3. Yang D-P, Kong D-X \& Zhang H-Y (2007) Multiple pharmacological effects of olive oil phenols. Food Chem $\mathbf{1 0 4}$ 1269-1271.

4. Aguilera CM, Mesa MD, Ramirez-Tortosa MC, et al. (2004) Sunflower oil does not protect against LDL oxidation as virgin olive oil does in patients with peripheral vascular disease. Clin Nutr 23, 673-681. 
5. Silva S, Gomes L, Leitao F, et al. (2006) Phenolic compounds and antioxidant activity of Olea europaea L. fruits and leaves. Food Sci Technol Int 12, 385-395.

6. Kountouri AM, Mylona A, Kaliora AC, et al. (2007) Bioavailability of the phenolic compounds of the fruits (drupes) of Olea europaea (olives): impact on plasma antioxidant status in humans. Phytomedicine 14, 659-667.

7. Owen RW, Mier W, Giacosa A, et al. (2000) Phenolic compounds and squalene in olive oils: the concentration and antioxidant potential of total phenols, simple phenols, secoiridoids, lignansand squalene. Food Chem Toxicol 38, 647-659.

8. Servili M \& Montedoro G (2002) Contribution of phenolic compounds to virgin olive oil quality. Eur J Lip Sci Technol 104, 602-613.

9. Leporatti ML, Posocco E \& Pavesi A (1985) Some new therapeutic uses of several medicinal plants in the province of Terni (Umbria, Central Italy). J Ethnopharmacol 14, 65-68.

10. Khayyal MT, Elghazaly MA, Abdallah DM, et al. (2002) Blood pressure lowering effect of an olive leaf extract (Olea europaea) in L-NAME induced hypertension in rats. Arzneimittelforschung (Drug Res) 52, 797-802.

11. Al-Azzawie HF \& Alhamdani M-SS (2006) Hypoglycemic and antioxidant effect of oleuropein in alloxan-diabetic rabbits. Life Sci 78, 1371-1377.

12. Miles EA, Zoubouli P \& Calder PC (2005) Differential antiinflammatory effects of phenolic compounds from extra virgin olive oil identified in human whole blood cultures. Nutrition 21, 389-394.

13. Wang L, Geng C, Jiang L, et al. (2008) The anti-atherosclerotic effect of olive leaf extract is related to suppressed inflammatory response in rabbits with experimental atherosclerosis. Eur J Nutr 47, 235-243.

14. Petkov VM \& Manolov P (1972) Pharmacological analysis of the iridoid oleuropein. Arzneimittelforschung (Drug Res) 22, 1476-1488.

15. Lockyer S, Yaqoob P, Spencer J, et al. (2012) Olive leaf phenolics and cardiovascular risk reduction: physiological effects and mechanisms of action. Nutr Aging 1, 125-140.

16. Ruano J, Lopez-Miranda J, Fuentes F, et al. (2005) Phenolic content of virgin olive oil improves ischemic reactive hyperemia in hypercholesterolemic patients. J Am Coll Cardiol 46, $1864-1868$.

17. Jiménez-Morales AI, Ruano J, Delgado-Lista J, et al. (2011) NOS3 Glu298Asp polymorphism interacts with virgin olive oil phenols to determine the postprandial endothelial function in patients with the metabolic syndrome. I Clin Endocrinol Metab 96, E1694-E1702.

18. Vasan RS (2006) Biomarkers of cardiovascular disease. Circulation 113, 2335-2362.

19. Camargo A, Ruano J, Fernandez J, et al. (2010) Gene expression changes in mononuclear cells in patients with metabolic syndrome after acute intake of phenol-rich virgin olive oil. BMC Genomics 11, 253.

20. Corona G, Spencer J \& Dessì M (2009) Extra virgin olive oil phenolics: absorption, metabolism, and biological activities in the GI tract. Toxicol Ind Health 25, 285-293.

21. Kendall M, Batterham M, Callahan DL, et al. (2012) Randomized controlled study of the urinary excretion of biophenols following acute and chronic intake of olive leaf supplements. Food Chem 130, 651-659.

22. de Bock M, Thorstensen EB, Derraik JGB, et al. (2013) Human absorption and metabolism of oleuropein and hydroxytyrosol ingested as olive (Olea europaea L.) leaf extract. Mol Nutr Food Res 5, 2079-2085.
23. Visioli F, Galli C, Grande S, et al. (2003) Hydroxytyrosol excretion differs between rats and humans and depends on the vehicle of administration. J Nutr 133, 2612-2615.

24. Williams MR, Westerman RA, Kingwell BA, et al. (2001) Variations in endothelial function and arterial compliance during the menstrual cycle. J Clin Endocrinol Metab 86, 5389-5395.

25. de Bock M, Derraik JG, Brennan CM, et al. (2013) Olive (Olea europaea L.) leaf polyphenols improve insulin sensitivity in middle-aged overweight men: a randomized, placebo-controlled, crossover trial. PLOS ONE 8, e57622.

26. Wykretowicz A, Gerstenberger P, Guzik P, et al. (2009) Arterial stiffness in relation to subclinical atherosclerosis. Eur J Clin Invest 39, 11-16.

27. Millasseau SCKR, Ritter JM \& Chowienczyk PJ (2002) Determination of age-related increases in large artery stiffness by digital pulse contour analysis. Clin Sci (Lond) 103, 371-377.

28. Pinto J, Paiva-Martins F, Corona G, et al. (2011) Absorption and metabolism of olive oil secoiridoids in the small intestine. Br J Nutr 105, 1607.

29. Malik AR, Kondragunta V \& Kullo IJ (2008) Forearm vascular reactivity and arterial stiffness in asymptomatic adults from the community. Hypertension 51, 1512-1518.

30. Gori T (2006) Olive oil and ischemic reactive hyperemia in hypercholesterolemic patients. J Am Coll Cardiol 48, 414.

31. Giugliano D \& Esposito K (2006) Virgin olive oil and vegetables improve endothelial health. J Am Coll Cardiol 48, 413-414.

32. Steffen Y, Gruber C, Schewe T, et al. (2008) Mono-O-methylated flavanols and other flavonoids as inhibitors of endothelial NADPH oxidase. Arch Biochem Biophys 469, 209-219.

33. Rodriguez-Mateos A, Rendeiro C, Bergillos-Meca T, et al. (2013) Intake and time dependence of blueberry flavonoidinduced improvements in vascular function: a randomized, controlled, double-blind, crossover intervention study with mechanistic insights into biological activity. Am J Clin Nutr 98, 1179-1191.

34. Stolk J, Hiltermann T, Dijkman J, et al. (1994) Characteristics of the inhibition of NADPH oxidase activation in neutrophils by apocynin, a methoxy-substituted catechol. Am J Respir Cell Mol Biol 11, 95-102.

35. Vissers MN, Zock PL, Roodenburg AJC, et al. (2002) Olive oil phenols are absorbed in humans. J Nutr 132, 409-417.

36. Corona G, Tzounis X, Assunta Dessì M, et al. (2006) The fate of olive oil polyphenols in the gastrointestinal tract: implications of gastric and colonic microflora-dependent biotransformation. Free Radic Res 40, 647-658.

37. Pinto J, Paiva-Martins F, Corona G, et al. (2011) Absorption and metabolism of olive oil secoiridoids in the small intestine. Br J Nutr 105, 1607-1618.

38. Marchesi S, Lupattelli G, Schillaci G, et al. (2000) Impaired flow-mediated vasoactivity during post-prandial phase in young healthy men. Atherosclerosis 153, 397-402.

39. Esposito K, Nappo F, Giugliano F, et al. (2003) Effect of dietary antioxidants on postprandial endothelial dysfunction induced by a high-fat meal in healthy subjects. Am J Clin Nutr 77, 139-143.

40. Nappo F, Esposito K, Cioffi M, et al. (2002) Postprandial endothelial activation in healthy subjects and in type 2 diabetic patients: role of fat and carbohydrate meals. $J \mathrm{Am}$ Coll Cardiol 39, 1145-1150.

41. van Oostrom AJ, Sijmonsma TP, Verseyden C, et al. (2003) Postprandial recruitment of neutrophils may contribute to endothelial dysfunction. J Lipid Res 44, 576-583. 
42. Petroni A, Blasevich M, Salami M, et al. (1995) Inhibition of platelet aggregation and eicosanoid production by phenolic components of olive oil. Thromb Res 78, 151-160.

43. Rizza L, Munafo S \& Serraino A (2012) Compositions for the treatment of hemorrhoids and related diseases. Google Patents.

44. Peveri P, Walz A, Dewald B, et al. (1988) A novel neutrophilactivating factor produced by human mononuclear phagocytes. J Exp Med 167, 1547-1559.

45. Frostegård J, Ulfgren A-K, Nyberg P, et al. (1999) Cytokine expression in advanced human atherosclerotic plaques: dominance of pro-inflammatory (Th1) and macrophagestimulating cytokines. Atherosclerosis 145, 33-43.

46. Shin WS, Szuba A \& Rockson SG (2002) The role of chemokines in human cardiovascular pathology: enhanced biological insights. Atherosclerosis 160, 91-102.

47. D'Agostino RB Sr, Pencina MJ, Massaro JM, et al. (2013) Cardiovascular disease risk assessment: insights from Framingham. Glob Heart 8, 11-23.
48. Boekholdt SM, Peters RJ, Hack CE, et al. (2004) IL-8 plasma concentrations and the risk of future coronary artery disease in apparently healthy men and women: the EPIC-Norfolk Prospective Population Study. Arterioscler Thromb Vasc Biol 24, 1503-1508.

49. DeForge LE, Fantone JC, Kenney JS, et al. (1992) Oxygen radical scavengers selectively inhibit interleukin 8 production in human whole blood. J Clin Invest 90, 2123.

50. Rahman I, Biswas SK \& Kirkham PA (2006) Regulation of inflammation and redox signaling by dietary polyphenols. Biochem Pharmacol 72, 1439-1452.

51. Carluccio MA, Siculella L, Ancora MA, et al. (2003) Olive oil and red wine antioxidant polyphenols inhibit endothelial activation: antiatherogenic properties of Mediterranean diet phytochemicals. Arterioscler Thromb Vasc Biol 23, 622-629.

52. Preedy VR \& Watson RR (2010) Olives and Olive Oil in Health and Disease Prevention. Amsterdam: Elsevier, Inc. 